\title{
Multidisciplinary consensus on the classification of antenatal and postnatal urinary tract dilation (UTD classification system)
}

\author{
Jeanne S. Chow • Kassa Darge
}

Received: 11 November 2014 /Revised: 10 December 2014 / Accepted: 4 February 2015 /Published online: 13 March 2015

(C) Springer-Verlag Berlin Heidelberg 2015

Approximately 40,000 to 50,000 children are diagnosed yearly with urinary tract dilation. Correlation between antenatal and postnatal US findings has been difficult due to the lack of consensus in defining and describing urinary tract dilation. There is variability not only among subspecialty groups, but also within the Society for Pediatric Radiology (SPR). A recent survey showed that pediatric radiologists have no standard method to describe urinary tract dilation. Some use a descriptive system (e.g., mild, moderate or severe) while others use standard grading systems (e.g., the Society of Fetal Urology's numerical grading system $0-4$ ). Ninety-five percent of SPR survey responders favor a standard system although the vast majority do not have a system to recommend [1].

Representatives from eight societies with interest in the diagnosis and management of fetuses and children with urinary tract dilation - the American College of Radiology (ACR), the American Institute of Ultrasound in Medicine (AIUM), the American Society of Pediatric Nephrology (ASPN), the Society for Fetal Urology (SFU), the Society for Maternal-Fetal Medicine (SMFM), the Society for Pediatric Urology (SPU), the SPR and the Society of Radiologists in Ultrasound

J. S. Chow $(\bowtie)$

Departments of Radiology and Urology, Boston Children's Hospital, 300 Longwood Ave., Boston, MA 02116, USA

e-mail: Jeanne.chow@childrens.harvard.edu

K. Darge

Department of Radiology, Children's Hospital of Philadelphia, Philadelphia, PA, USA
(SRU) - agreed to collaborate on the development of a standard grading system for urinary tract dilation and make recommendations for follow-up evaluation. The consensus conference took place on March 14-15, 2014, in Linthicum, MD.

The Consensus Panel proposed the UTD (Urinary Tract Dilation) Classification System to describe the appearance of the urinary tract both antenatally and postnatally. Recommendations for further evaluation of these patients were made based on the UTD grade. This new system is expected to be validated and/or modified with clinical experience and future research.

The proposed UTD Classification System (Fig. 1) is based on six US findings: 1) anterior-posterior renal pelvic diameter (APRPD), 2) calyceal dilation with distinction between central and peripheral calyceal dilation postnatally, 3) renal parenchymal thickness, 4) renal parenchymal appearance, 5) bladder abnormalities and 6) ureteral abnormalities. For antenatal studies, the seventh US finding to report is the quantity of amniotic fluid. Normal values are also defined.

The UTD Classification System distinguishes whether findings are antenatal ("A") or postnatal ("P"). The higher the number, the more severe the findings. Thus, UTD A1 represents mild antenatal urinary tract dilation and UTD P3 represents severe urinary tract dilation and/or other abnormalities such as renal hyperechogenicity or a thick bladder wall after birth. Grading is based on the most severe finding. Because abnormalities of the urinary tract are more subtle and difficult to visualize in a fetus than in a child, there are only three antenatal categories (Normal, UTD A1, UTD A2-3) and four postnatal catagories (Normal, UTD P1, UTD P2 and UTD P3).

Due to the great confusion associated with the implied meanings of various terminologies for urinary 


\section{UTD Classification System}

\section{Antenatal Presentation}
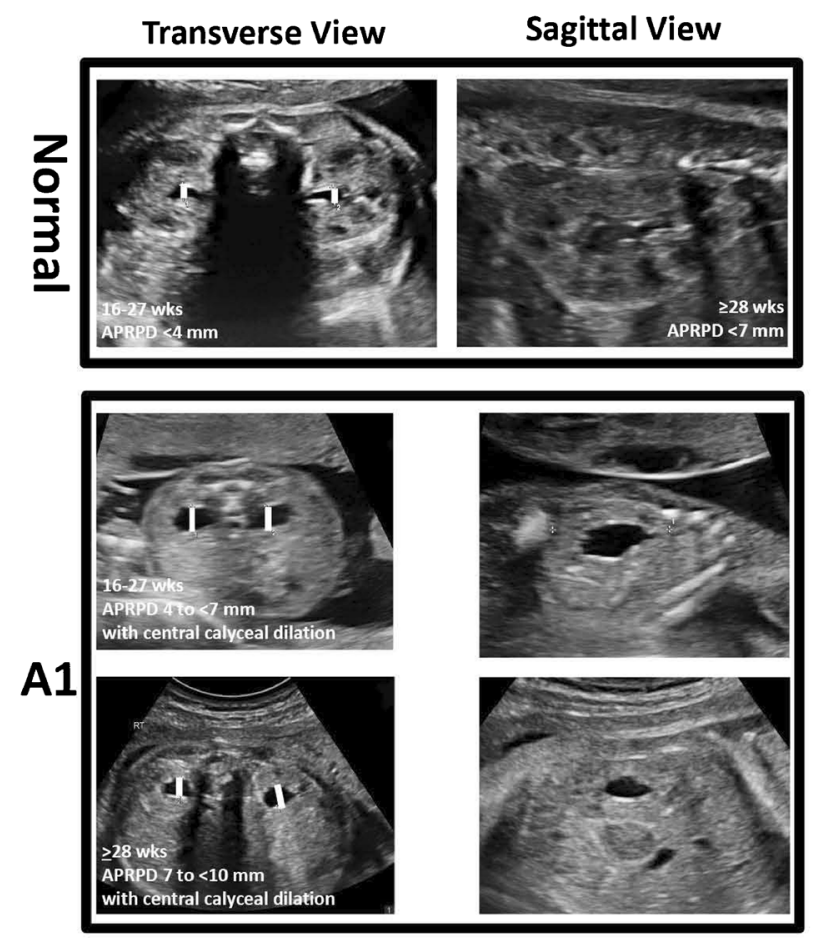

A2-3
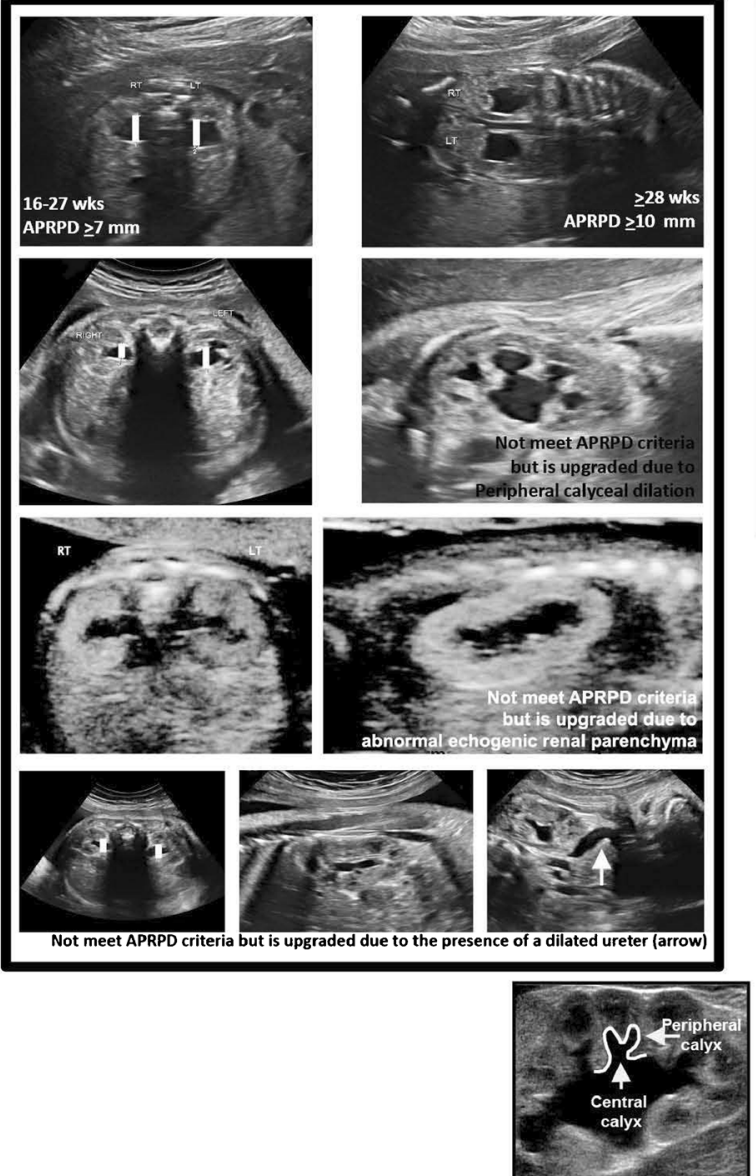

Postnatal Presentation Transverse View Sagittal View
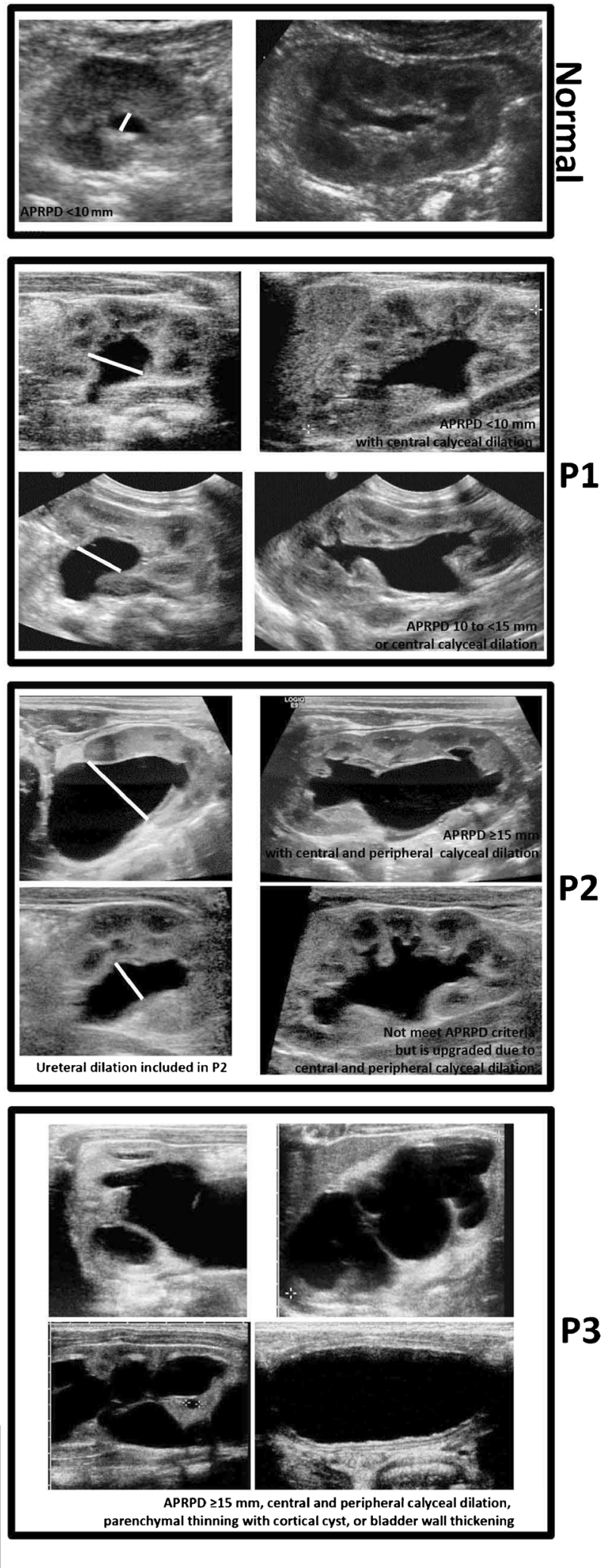
4 Fig. 1 The Urinary Tract Dilation (UTD) Classification System describes the appearance of the urinary tract based on antenatal and postnatal presentation. The Antenatal Presentation (left column) is divided into normal, A1 and A2-3, and measurements between 1627 weeks' and $\geq 28$ weeks' gestation. A normal urinary tract is one with no urinary tract abnormalities and anterior posterior renal pelvic diameter (APRPD) measuring less than $4 \mathrm{~mm}$ between 16-27 weeks' gestation and less than $7 \mathrm{~mm} \geq 28$ weeks' gestation. A1 describes a normal urinary tract with 4 to $<7 \mathrm{~mm}$ pelvic dilation at 16-27 weeks' gestation or 7 to $<10 \mathrm{~mm} \geq 28$ weeks' gestation with or without central calyceal dilation. UTD A2-3 is for fetuses if there is APRPD $\geq 7 \mathrm{~mm}$ between 16-27 weeks' gestation or $\geq 10 \mathrm{~mm} \geq 28$ weeks' gestation, peripheral calyceal dilation, ureteral dilation, renal parenchymal or bladder abnormalities. The Postnatal Presentation (right column) is divided into normal, P1, P2 and P3. Measurements are more reliable if taken $48 \mathrm{~h}$ after birth or later. A normal urinary tract is one with no urinary tract abnormalities and APRPD $<10 \mathrm{~mm}$. P1 describes a normal urinary tract with APRPD 10 to $<15 \mathrm{~mm}$ and/or central calyceal dilation. P2 describes APRPD $\geq 15 \mathrm{~mm}$ or peripheral calyceal dilation. P3 describes additional ureteral dilation, abnormal renal echogenicity or cysts or bladder abnormalities regardless of APRPD measurement.

dilation, the Consensus Panel recommends consistent use of the term "dilation" and avoiding the use of non-specific terms such as hydronephrosis, pyelectasis, pelviectasis or pelvic fullness.

When reporting urinary tract dilation, a description of the above six imaging parameters should be described in the body of the report. The specific UTD category (Normal, UTD A1, UTD A2-3, UTD P1, UTD P2 or UTD P3) should be in the impression.

The UTD Classification System and the recommendations of the Consensus Panel are published in the Journal of Pediatric Urology [2]. We hope that the proposed recommendations will help to decrease confusion and to standardize the care of fetuses and children with urinary tract dilation and facilitate future research. Pediatric radiologists want a unified system to describe urinary tract dilation and we strongly believe that the UTD Classification System is the answer. Pediatric radiologists should adopt this system.

Conflicts of interest None

\section{References}

1. Swenson DW, Darge K, Ziniel SI et al (2014) Characterizing upper urinary tract dilation on ultrasound: a survey of North American pediatric radiologists' practices. Pediatr Radiol. doi:10.1007/s00247-0143221-8

2. Nguyen HT, Benson CB, Bromley B et al (2014) Multidisciplinary consensus on the classification of antenatal and postnatal urinary tract dilation (UTD Classification System). J Pediatr Urol 10:982-998 